\title{
A theory for the isolation of the complex deformation characteristics of nonlinear viscoelastic materials
}

\author{
D. W. Holmes ${ }^{1} \quad$ J. G. Loughran ${ }^{2}$
}

(Received 31 August 2006; revised 13 December 2007)

\begin{abstract}
This article reports on the development of a new numerical method for the separation of multiple interdependent nonlinear deformation phenomena. Whilst conceivably applicable to other classes on nonlinear materials, the method proposed was developed specifically for large strain nonlinear viscoelasticity. Characterized by a combination of elastic and viscous deformation contributions, the corresponding constitutive relationships become experimentally indeterminate when both components of response are nonlinearly interdependent. The theory presented here incorporates a methodology analogous to Closest Point Projection of computational plasticity which is used like a Newton type iterative solution for multiple, mutually dependent
\end{abstract}

See http://anziamj.austms.org.au/ojs/index.php/ANZIAMJ/article/view/119 for this article, (c) Austral. Mathematical Soc. 2007. Published December 19, 2007. ISSN 1446-8735 
parameters. The proposed technique is shown to closely reproduce the results seen from numerical simulations given prescribed nonlinear elastic and viscous relationships. This is of particular benefit to the accurate determination of material model coefficients.

\section{Contents}

1 Introduction

C646

2 Constitutive equations

C647

3 Parameter extraction techniques

C649

4 Theoretical development

C650

5 Numerical example

C655

6 Conclusion

C658

References

C658

\section{Introduction}

A major limitation of complex constitutive theories arises from the extraction of model parameters from laboratory testing needed to allow meaningful implementation. For hyperelastic materials such as rubbers, relatively standardised parameter determination techniques have been developed using optimisation algorithms such as that of Levenberg and Marquardt [11, for example]. In such cases the total material test stress is related to total test strain through the fit of a parametrised modulus. However, analogous techniques for cases where inelastic evolution is present are largely absent from 
the literature. For a single Maxwell type viscoelastic element [9], in the one dimensional case, a split of strain into elastic and inelastic components gives

$$
\varepsilon=\varepsilon_{e}+\varepsilon_{i}
$$

The elastic modulus $E$ is then related to total stress $\sigma$ and the elastic component of strain $\varepsilon_{e}$ by

$$
\sigma=E \varepsilon_{e},
$$

while the viscosity $\eta$ is related to the total stress $\sigma$ and the inelastic component of strain rate $\dot{\varepsilon}_{i}$ by

$$
\dot{\varepsilon}_{i}=\frac{1}{\eta} \sigma .
$$

This presents a problem resulting from the restrictions of standard tensile test techniques. Through component isolation techniques (Brusselle-Dupend et al. $[3,4])$ it is possible to output from testing the viscoelastic element stress and the total element strain. However, given such data, from the current form of (1), (2) and (3), it is not possible to solve simultaneously for the parameters $E$ and $\eta$ on a point by point basis. In addition, if $E$ and $\eta$ have functional dependencies and three dimensionality, direct solution cannot be achieved and so some iterative solution is required.

We present a new iterative approach to the calculation of model parameters for 3D nonlinear, time dependent, materials paying particular attention to viscoelasticity.

\section{Constitutive equations}

Thermodynamic continuum theory stems from the approximation of thermodynamic free energy function, dependent on the current state of strain and a set of globally applicable material parameters. Such a function is often expressed in principal space with decoupled volumetric and deviatoric 
components (Simo and Taylor [10]),

$$
\psi\left(\varepsilon_{1 e}, \varepsilon_{2 e}, \varepsilon_{3 e}\right)=\underbrace{U\left(J_{e}\right)}_{\text {vol }}+\underbrace{\tilde{w}\left(\epsilon_{1 e}, \epsilon_{2 e}, \epsilon_{3 e}\right)}_{\text {dev }},
$$

where $\varepsilon_{A e}$ and $\epsilon_{A e}$ are total and deviatoric logarithmic principal elastic strains while $J_{e}=\exp \left(\varepsilon_{1 e}\right) \exp \left(\varepsilon_{2 e}\right) \exp \left(\varepsilon_{3 e}\right)$ is principal volumetric strain.

The principal Kirchhoff stress is then defined by the differential

$$
\tau_{A}=\frac{\partial \psi\left(\varepsilon_{1 e}, \varepsilon_{2 e}, \varepsilon_{3 e}\right)}{\partial \varepsilon_{A e}}=\underbrace{\frac{\partial U\left(J_{e}\right)}{\partial J_{e}} \frac{\partial J_{e}}{\partial \varepsilon_{A e}}}_{\text {vol }}+\underbrace{\sum_{B=1}^{3} \frac{\partial \tilde{w}\left(\epsilon_{1 e}, \epsilon_{2 e}, \epsilon_{3 e}\right)}{\partial \epsilon_{B e}} \frac{\partial \epsilon_{B e}}{\partial \varepsilon_{A e}}}_{\text {dev }} .
$$

The 3D continuum theory analogue of the elastic relationship (2), now takes a more complex form. Here any incremental increase in stress is a function of both the corresponding increase in strain as well as the current total state of strain. Using the volumetric and deviatoric components of Kirchhoff stress from (5)

$$
\frac{\partial \tau_{A}}{\partial \varepsilon_{B e}}=\frac{\partial \operatorname{vol}\left[\tau_{A}\right]}{\partial J_{e}} \frac{\partial J_{e}}{\partial \varepsilon_{B e}}+\sum_{C=1}^{3} \frac{\partial \operatorname{dev}\left[\tau_{A}\right]}{\partial \epsilon_{C e}} \frac{\partial \epsilon_{C e}}{\partial \varepsilon_{B e}},
$$

which is the complete $3 \mathrm{D}$ elastic moduli.

For cases where inelasticity is present, the total strain has elastic and inelastic components. In the case of nonlinear viscoelasticity, suitable treatment of inelastic evolution is presented by Reese and Govindjee [8]

$$
\vec{\varepsilon}_{e}=\vec{\varepsilon}_{e}^{\text {trial }}-\frac{\Delta t}{2}\left[\boldsymbol{I}_{3} \vec{\eta}\right]^{-1} \operatorname{dev}[\vec{\tau}] .
$$

This expression ${ }^{1}$ represents the correction in a step in principal elastic strain required to account for inelastic evolution following an initial trial esti-

${ }^{1}$ Note $\mathbf{1}=\delta_{i}, \boldsymbol{I}=\delta_{i j}$ and $\boldsymbol{I}_{3}=\delta_{i j k}, i, j, k=1,2,3$ denote 1st, 2nd and 3rd order Kronecker delta tensors as explained, for example, by Bonet and Wood [2] or Simo and Hughes [9]. 
mate $\vec{\varepsilon}_{e}^{\text {trial }}$ assuming the current step in strain to have been wholly elastic. The term $\vec{\eta}$ is the principal viscosity vector with a possible nonlinear functionality on rate.

(7) infers that the inelastic component of strain is wholly deviatoric. This is a common simplification which is practical in many real circumstances [8].

This group of constitutive expressions presents an excellent test case for an iterative algorithm to isolate nonlinear material parameters. Given the intention of fitting such a model to experimental data, principal Kirchhoff stress and total logarithmic strain are known and several limitations are present. The elastic relationship (6) is assumed nonlinear and dependent on the elastic strain and some set of parameters that must fit the whole set of data. From the evolution (7), while the viscosity coefficient itself is assumed to have unknown dependence on rate, providing elastic strain and stress are known, this value can be calculated directly for each data point. This discontinuity between global and local fit of parameters poses significant difficulties.

\section{Parameter extraction techniques}

Implementation of a complex, multi-nonlinear theory based on thermodynamic principles is rarely carried out in industrial circumstances due to parameter generation difficulties. Less complex theories are more commonplace, making use of one or more simplifications to increase practicality.

Reese and Govindjee [8] present a viscoelastic model with Ogden hyperelasticity and constant viscosity for application to various rubbers. Constant viscosity greatly simplifies parameter calculation.

Brusselle-Dupend et al. $[3,4]$ present research on a semicrystalline polymer response, observing the viscoelastic viscosity to be highly dependent on 
elastic and inelastic strain rates. To allow calculation of the nonlinear viscosity curve, they assume a linear relationship between stress and elastic strain and calculate viscosity by rearrangement of an expression similar to (7).

An advantage to a theory that allows pointwise calculation of all nonlinear values is that the dependencies of each need not be explicitly defined before hand. Such a property is of significant benefit, particularly within materials research on new or complexly dependent materials where such dependencies are unknown. Here we present a theoretical development of such a theory which is applied to the case of nonlinear viscoelasticity that is not limited by linear assumptions or pre-specified functional dependencies.

\section{Theoretical development}

In order to completely define the material model outlined in Section 2 from experimental test results, it would be desirable to be able to calculate elastic strain $\vec{\varepsilon}_{e}$ and viscosity $\vec{\eta}$ for all data points. It would then be a trivial extension to apply least squares curve fitting techniques to fit suitable elasticity and viscosity functions to the calculated values.

We begin by noting that the inelastic strain, $\vec{\varepsilon}_{i}$ is wholly deviatoric. Hence $\operatorname{vol}\left[\vec{\varepsilon}_{e}\right]=\operatorname{vol}[\vec{\varepsilon}]$ and thus $J_{e}\left(\vec{\varepsilon}_{e}\right)=J(\vec{\varepsilon})$. As a result the volumetric terms of (5) and (6) become invariant to inelastic evolution and so need not be considered in what follows. Subsequently the deviatoric stress $\operatorname{dev}[\vec{\tau}]$ is written as the simpler $\vec{\tau}$.

The linearized form of the modulus expression (6) is

$$
\partial \vec{\tau}=\left[\frac{\partial \vec{\tau}}{\partial \vec{\epsilon}_{e}} \frac{\partial \vec{\epsilon}_{e}}{\partial \vec{\varepsilon}_{e}}\right] \partial \vec{\varepsilon}_{e}=\boldsymbol{C} \partial \vec{\varepsilon}_{e},
$$

where $\boldsymbol{C}$ is the 2 nd order deviatoric modulus tensor. Ideally multiplication of (8) by $\boldsymbol{C}^{-1}$ should result in $\partial \vec{\varepsilon}_{e}$ however the volumetric component of $\boldsymbol{C}$ 
does not contain sufficient information to do this because of the whole form of the expression, (6).

To counter this, we draw attention to the $\partial \vec{\epsilon}_{e} / \partial \vec{\varepsilon}_{e}$ term. The deviatoric strain is defined by

$$
\vec{\epsilon}_{e}=\vec{\varepsilon}_{e}-\frac{1}{3} \ln J_{e} \mathbf{1}
$$

Carrying out the differentiation

$$
\frac{\partial \epsilon_{e A}}{\partial \varepsilon_{e B}}=\frac{\partial}{\partial \varepsilon_{e B}}\left(\varepsilon_{e A}-\frac{1}{3} \ln J_{e} 1_{A}\right)=\delta_{A B}-\frac{1}{3} \delta_{A} \otimes \delta_{B},
$$

where $A, B=1,2,3$.

By exploiting $\partial \vec{\epsilon}_{e} / \partial \vec{\varepsilon}_{e}$ while inverting (8), a consistent result is achieved

$$
\frac{\partial \vec{\epsilon}_{e}}{\partial \vec{\varepsilon}_{e}} \boldsymbol{C}^{-1} \partial \vec{\tau}=\frac{\partial \vec{\epsilon}_{e}}{\partial \vec{\varepsilon}_{e}} \partial \vec{\varepsilon}_{e}=\partial \vec{\epsilon}_{e} .
$$

Now focussing on (7), $\vec{\varepsilon}_{e}^{\text {trial }}$ is derived from operator split methodology [5] such that for time $t=t_{n+1}$

$$
\vec{\varepsilon}_{e n+1}^{\text {trial }}=\vec{\varepsilon}_{e n}+\left(\vec{\varepsilon}_{n+1}-\vec{\varepsilon}_{n}\right) .
$$

Applying this and (9) to the strain terms in (7) and because $J_{e}=J$

$$
\begin{aligned}
\vec{\varepsilon}_{e n+1}-\vec{\varepsilon}_{e n+1}^{\text {trial }}= & \vec{\epsilon}_{e n+1}+\frac{1}{3} \ln J_{e n+1} \mathbf{1}-\vec{\epsilon}_{e n}-\frac{1}{3} \ln J_{e n} \mathbf{1} \\
& -\vec{\epsilon}_{n+1}-\frac{1}{3} \ln J_{n+1} \mathbf{1}+\vec{\epsilon}_{n}+\frac{1}{3} \ln J_{n} \mathbf{1} \\
= & \vec{\epsilon}_{e n+1}-\vec{\epsilon}_{e n+1}^{\text {trial }}
\end{aligned}
$$

where now

$$
\vec{\epsilon}_{e n+1}^{\text {trial }}=\vec{\epsilon}_{e n}+\left(\vec{\epsilon}_{n+1}-\vec{\epsilon}_{n}\right)
$$

Now reformulate (7) with respect to deviatoric strain components as

$$
\vec{\epsilon}_{e n+1}=\vec{\epsilon}_{e n+1}^{\text {trial }}-\frac{\Delta t}{2}\left[\boldsymbol{I}_{3} \vec{\eta}_{n+1}\right]^{-1} \operatorname{dev}\left[\vec{\tau}_{n+1}\right] .
$$


Equations (8) (with substitution of (9)), (11) and (15) are unique and functional on the three mutually dependent values $\boldsymbol{C}, \vec{\epsilon}_{e}$ and $\vec{\eta}$. A convenient manipulation is to substitute (11) into (15) to give a different form of the equation set:

$$
\begin{aligned}
\Delta t \frac{\partial \vec{\epsilon}_{e}}{\partial \vec{\varepsilon}_{e}} \boldsymbol{C}_{n+1}^{-1} \dot{\vec{\tau}}_{n+1}+\vec{\epsilon}_{e n}-\vec{\epsilon}_{e n+1}^{\text {trial }} & =-\frac{\Delta t}{2}\left[\boldsymbol{I}_{3} \vec{\eta}_{n+1}\right]^{-1} \vec{\tau}_{n+1} \\
& =-\frac{\Delta t}{2} \boldsymbol{I}_{3}\left[\boldsymbol{I}_{3} \vec{\eta}_{n+1}\right]^{-1} \vec{\tau}_{n+1} \mathbf{1} \\
& =-\frac{\Delta t}{2} \boldsymbol{I}_{3} \vec{\tau}_{n+1}\left[\boldsymbol{I}_{3} \vec{\eta}_{n+1}\right]^{-1} \mathbf{1} .
\end{aligned}
$$

Rearranging $^{2}$

$$
\left\{\begin{array}{l}
\eta_{1 n+1}^{-1} \\
\eta_{2 n+1}^{-1} \\
\eta_{3 n+1}^{-1}
\end{array}\right\}=-\frac{2}{\Delta t}\left[\boldsymbol{I}_{3} \vec{\tau}_{n+1}\right]^{-1}\left\{\Delta t \frac{\partial \vec{\epsilon}_{e}}{\partial \vec{\varepsilon}_{e}} \boldsymbol{C}_{n+1}^{-1} \dot{\vec{\tau}}_{n+1}+\vec{\epsilon}_{e n}-\vec{\epsilon}_{e n+1}^{\text {trial }}\right\}
$$

Because there are three expressions and three mutually dependent variables, a solution cannot be directly achieved by standard iterative methods such as Newton's method. The key to such an operation can be found within plastic and viscoplastic theory [7,9] whereby the method referred to as Closest Point Projection (CPP) becomes an analogue to a multiple term, Newton's method when more than two mutually dependant values are present. Correspondingly, Equations (8) (with substitution of (9)), (15) and now (16) are rearranged into residual functions to be minimized $^{3}$

$$
\begin{aligned}
r_{\vec{\epsilon}_{e}} & =\vec{\epsilon}_{e n+1}-\vec{\epsilon}_{e n+1}^{\text {trial }}+\frac{\Delta t}{2}\left[\boldsymbol{I}_{3} \vec{\eta}_{n+1}\right]^{-1} \vec{\tau}_{n+1}, \\
r_{\vec{\eta}} & =\overrightarrow{\left(\eta_{n+1}^{-1}\right)}+\frac{2}{\Delta t}\left[\boldsymbol{I}_{3} \vec{\tau}_{n+1}\right]^{-1}\left\{\Delta t \frac{\partial \vec{\epsilon}_{e}}{\partial \vec{\varepsilon}_{e}} \boldsymbol{C}_{n+1}^{-1} \dot{\vec{\tau}}_{n+1}+\vec{\epsilon}_{e n}-\vec{\epsilon}_{e n+1}^{\text {trial }}\right\},
\end{aligned}
$$

${ }^{2}$ When inelastic strain is isotropic, $\eta_{1}=\eta_{2}=\eta_{3}$; however, it is useful for what follows to keep the viscosity in vector form.

${ }^{3}$ In some highly nonlinear cases, the condition $\eta_{1}=\eta_{2}=\eta_{3}$ may have to be rigidly enforced which is done by including an extra residual function. 


$$
r_{\boldsymbol{C}}=\boldsymbol{C}_{n+1}:\left(\vec{\epsilon}_{e n+1}+\frac{1}{3} \ln J_{n+1} \mathbf{1}-\vec{\epsilon}_{e n}-\frac{1}{3} \ln J_{n} \mathbf{1}\right)-\Delta t \dot{\vec{\tau}}_{n+1} .
$$

The vector of inverse viscosity has been abbreviated in vector notation. Deriving each residual function with respect to their associated bases

$$
\begin{aligned}
\frac{\partial r_{\vec{\epsilon}_{e}}}{\partial \vec{\epsilon}_{e}} & =\boldsymbol{I}-\frac{\Delta t}{2}\left[\boldsymbol{I}_{3} \vec{\eta}_{n+1}\right]^{-2} \boldsymbol{I}_{3} \vec{\tau}_{n+1} \cdot \frac{\partial \vec{\eta}}{\partial \vec{\epsilon}_{e}}, \\
\frac{\partial r_{\vec{\eta}}}{\partial \vec{\eta}} & =-\boldsymbol{I}_{3} \overrightarrow{\left(\eta_{n+1}^{-2}\right)}-\frac{2}{\Delta t}\left[\boldsymbol{I}_{3} \vec{\tau}_{n+1}\right]^{-1} \Delta t \frac{\partial \vec{\epsilon}_{e}}{\partial \vec{\varepsilon}_{e}} \boldsymbol{C}_{n+1}^{-2} \boldsymbol{I}_{4} \dot{\vec{\tau}}_{n+1}: \frac{\partial \boldsymbol{C}}{\partial \vec{\eta}}, \\
\frac{\partial r_{\boldsymbol{C}}}{\partial \boldsymbol{C}} & =\boldsymbol{I}_{4}:\left(\vec{\epsilon}_{e n+1}+\frac{1}{3} \ln J_{n+1} \mathbf{1}-\vec{\epsilon}_{e n}-\frac{1}{3} \ln J_{n} \mathbf{1}\right)+\boldsymbol{C}_{n+1} \frac{\partial \vec{\epsilon}_{e}}{\partial \boldsymbol{C}},
\end{aligned}
$$

where $I_{4 i j k l}=\frac{1}{2}\left(\delta_{i k} \delta_{j l}+\delta_{i l} \delta_{j k}\right)$ is the symmetric 4 th order identity tensor.

Linearizing the expressions by multiplying by the $\partial$ base values and noting that the change in residual function $\partial r$ is desired to correct $r$ toward zero, in other words $r+\partial r=0$ and thus $\partial r=-r$ and therefore

$$
\begin{aligned}
-r_{\vec{\epsilon}_{e}} & =\boldsymbol{I} \partial \vec{\epsilon}_{e}-\frac{\Delta t}{2}\left[\boldsymbol{I}_{3} \vec{\eta}_{n+1}\right]^{-2} \boldsymbol{I}_{3} \vec{\tau}_{n+1} \cdot \partial \vec{\eta}+\mathbf{0}_{3}: \partial \boldsymbol{C}, \\
-r_{\vec{\eta}} & =\mathbf{0} \partial \vec{\epsilon}_{e}-\boldsymbol{I}_{3} \overrightarrow{\left(\eta_{n+1}^{-2}\right)} \partial \vec{\eta}-\frac{2}{\Delta t}\left[\boldsymbol{I}_{3} \vec{\tau}_{n+1}\right]^{-1} \Delta t \frac{\partial \vec{\epsilon}_{e}}{\partial \vec{\varepsilon}_{e}} \boldsymbol{C}_{n+1}^{-2} \boldsymbol{I}_{4} \dot{\vec{\tau}}_{n+1}: \partial \boldsymbol{C}, \\
-r_{\boldsymbol{C}} & =\boldsymbol{C}_{n+1} \partial \vec{\epsilon}_{e}+\mathbf{0} \partial \vec{\eta}+\boldsymbol{I}_{4}:\left(\vec{\epsilon}_{e n+1}+\frac{1}{3} \ln J_{n+1} \mathbf{1}-\vec{\epsilon}_{e n}-\frac{1}{3} \ln J_{n} \mathbf{1}\right): \partial \boldsymbol{C} .
\end{aligned}
$$

Common practice in CPP operations is to combine (19) into one composite matrix expression that can be inverted to calculate the respective update values $\left(\partial \vec{\epsilon}_{e}, \partial \vec{\eta}\right.$ and $\left.\partial \boldsymbol{C}\right)$. For this composition to be achieved the right hand 3rd to 2nd order tensor contraction terms in (19) must be reworked so as to have an equivalent form in the 2nd order environment. A 3rd to 2nd order tensor contraction proceeds [2]

$$
\sum_{i, j, k=1}^{3} A_{i j k} e_{i} \otimes e_{j} \otimes e_{k}: \sum_{l, m=1}^{3} B_{l m} e_{l} \otimes e_{m}=\sum_{i, j, k=1}^{3} A_{i j k} B_{j k} e_{i} .
$$




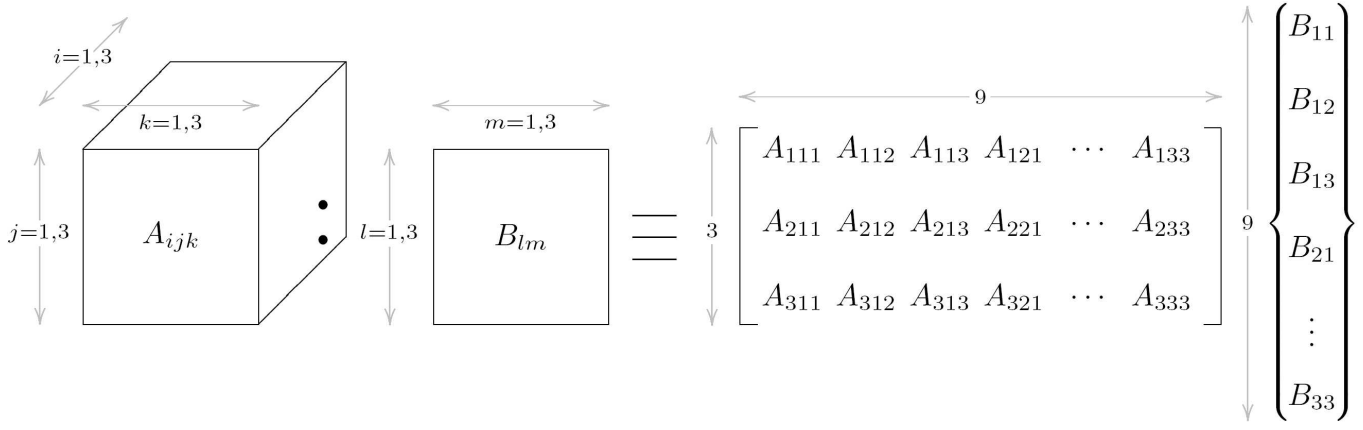

FiguRE 1: diagram illustrating equivalent 2nd order representation of 3rd to 2nd order tensor contraction.

The same vector product as in (20) is achieved by a non-square matrix to vector multiplication, as illustrated in Figure 1.

With this in mind, (19) is rewritten as

$$
\left[\begin{array}{lll}
\boldsymbol{\Xi}_{n+1}^{\epsilon \epsilon} & \boldsymbol{\Xi}_{n+1}^{\epsilon \eta} & \boldsymbol{\Xi}_{n+1}^{\epsilon C} \\
\boldsymbol{\Xi}_{n+1}^{\eta \epsilon} & \boldsymbol{\Xi}_{n+1}^{\eta \eta} & \boldsymbol{\Xi}_{n+1}^{\eta+} \\
\boldsymbol{\Xi}_{n+1}^{C \epsilon} & \boldsymbol{\Xi}_{n+1}^{C \eta} & \boldsymbol{\Xi}_{n+1}^{C C}
\end{array}\right]\left\{\begin{array}{c}
\partial \vec{\epsilon}_{e} \\
\partial \vec{\eta} \\
\partial \boldsymbol{C}
\end{array}\right\}=-\left\{\begin{array}{c}
r_{\vec{\epsilon}_{e}} \\
r_{\vec{\eta}} \\
r_{C}
\end{array}\right\} \equiv \boldsymbol{\Xi}_{n+1} \cdot \Delta=-\boldsymbol{R},
$$

where

$$
\begin{aligned}
& \boldsymbol{\Xi}_{n+1}^{\epsilon \epsilon}=\boldsymbol{I}, \\
& \boldsymbol{\Xi}_{n+1}^{\epsilon \eta}=-\frac{\Delta t}{2}\left[\boldsymbol{I}_{3} \vec{\eta}_{n+1}\right]^{-2} \boldsymbol{I}_{3} \vec{\tau}_{n+1}, \\
& \boldsymbol{\Xi}_{n+1}^{\epsilon C}=\mathbf{0}_{3}, \\
& \boldsymbol{\Xi}_{n+1}^{\eta \epsilon}=\mathbf{0}, \\
& \boldsymbol{\Xi}_{n+1}^{\eta \eta}=-\boldsymbol{I}_{3} \overrightarrow{\left(\eta_{n+1}^{-2}\right)}, \\
& \boldsymbol{\Xi}_{n+1}^{\eta C}=-\frac{2}{\Delta t}\left[\boldsymbol{I}_{3} \vec{\tau}_{n+1}\right]^{-1} \Delta t \frac{\partial \vec{\epsilon}_{e}}{\partial \vec{\varepsilon}_{e}} \boldsymbol{C}_{n+1}^{-2} \boldsymbol{I}_{4} \dot{\vec{\tau}}_{n+1}, \\
& \boldsymbol{\Xi}_{n+1}^{C \epsilon}=\boldsymbol{C}_{n+1},
\end{aligned}
$$




$$
\begin{aligned}
& \boldsymbol{\Xi}_{n+1}^{C \eta}=\mathbf{0}, \\
& \boldsymbol{\Xi}_{n+1}^{C C}=\boldsymbol{I}_{4}:\left(\vec{\epsilon}_{e n+1}+\frac{1}{3} \ln J_{n+1} \mathbf{1}-\vec{\epsilon}_{e n}-\frac{1}{3} \ln J_{n} \mathbf{1}\right),
\end{aligned}
$$

and where $\boldsymbol{\Xi}^{\epsilon C}, \boldsymbol{\Xi}^{\eta C}$ and $\boldsymbol{\Xi}^{C C}$ are $3 r d$ order tensors written into $3 \times 9$ matrices and $\partial \boldsymbol{C}$ is a 2 nd order tensor written as a $9 \times 1$ vector as per Figure 1 .

Calculating the inverse of $\boldsymbol{\Xi}$ in (21) gives the following expression where it becomes possible to determine the increments in strain, viscosity and modulus,

$$
\Delta=-\boldsymbol{\Xi}_{n+1}^{-1} \cdot \boldsymbol{R}
$$

A complexity arises in the calculation of $\boldsymbol{\Xi}^{-1}$ because it is non-square and rank deficient. As a result, no unique inverse exists. Applications such as this have motivated the formulation of a generalized theory (also called a Pseudoinverse) for partially inverting such matrices [1, 6, for example].

For this case $m<n$ for $\boldsymbol{\Xi}_{m n}$ which is insufficiently dimensioned for an unique solution to $\Delta$, thus the pseudoinverse operation corresponds to the minimization of the 2 -norm of $\Delta$, or in other words $\|\Delta\| \longrightarrow$ min. For cases where $m>n$, solution of the over dimensioned state corresponds to the least squares minimization of (21).

Iterative implementation of (22) allows the values of $\vec{\eta}, \vec{\epsilon}_{e}$ and $\boldsymbol{C}$ to be obtained on a pointwise basis and invariant to any explicit expression of elasticity or viscosity.

\section{Numerical example}

Meaningful verification of the proposed method requires a predetermined knowledge of the results to be calculated. As discussed, this is not possible using experimental results from conventional finite strain techniques. As a consequence a numerical test implementation of the viscoelastic material 


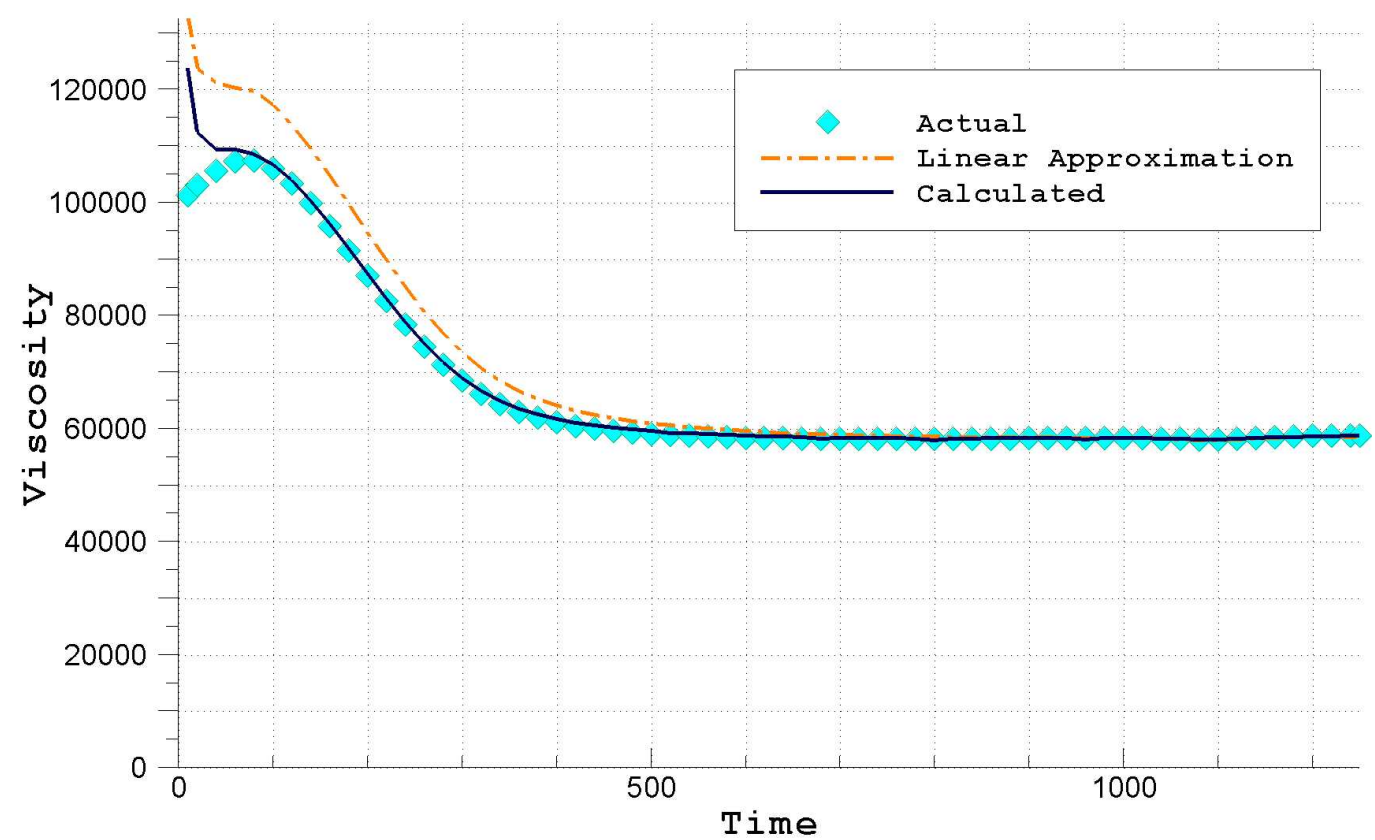

Figure 2: Plot showing viscosity against time.

model presented in Section 2 was solved with prescribed nonlinear elastic and viscous relationships to validate the performance of the proposed technique.

A uniaxial test simulation was carried out to $10 \%$ strain at a strain rate of $8 \times 10^{-5} / \mathrm{s}$ (as per polypropylene testing of Brusselle-Dupend et al. [3, 4]) and triaxial deviatoric stress and total logarithmic strain was output as would be possible from actual laboratory experiments.

The viscosity against time and elastic strain against time simulation results along with the performance of the proposed algorithm are shown in Figures 2 and 3 respectively. Also shown in these figures are approximations based on the assumption of linear stress-strain relationship in line with the methods of Brusselle-Dupend et al. [3, 4]. 


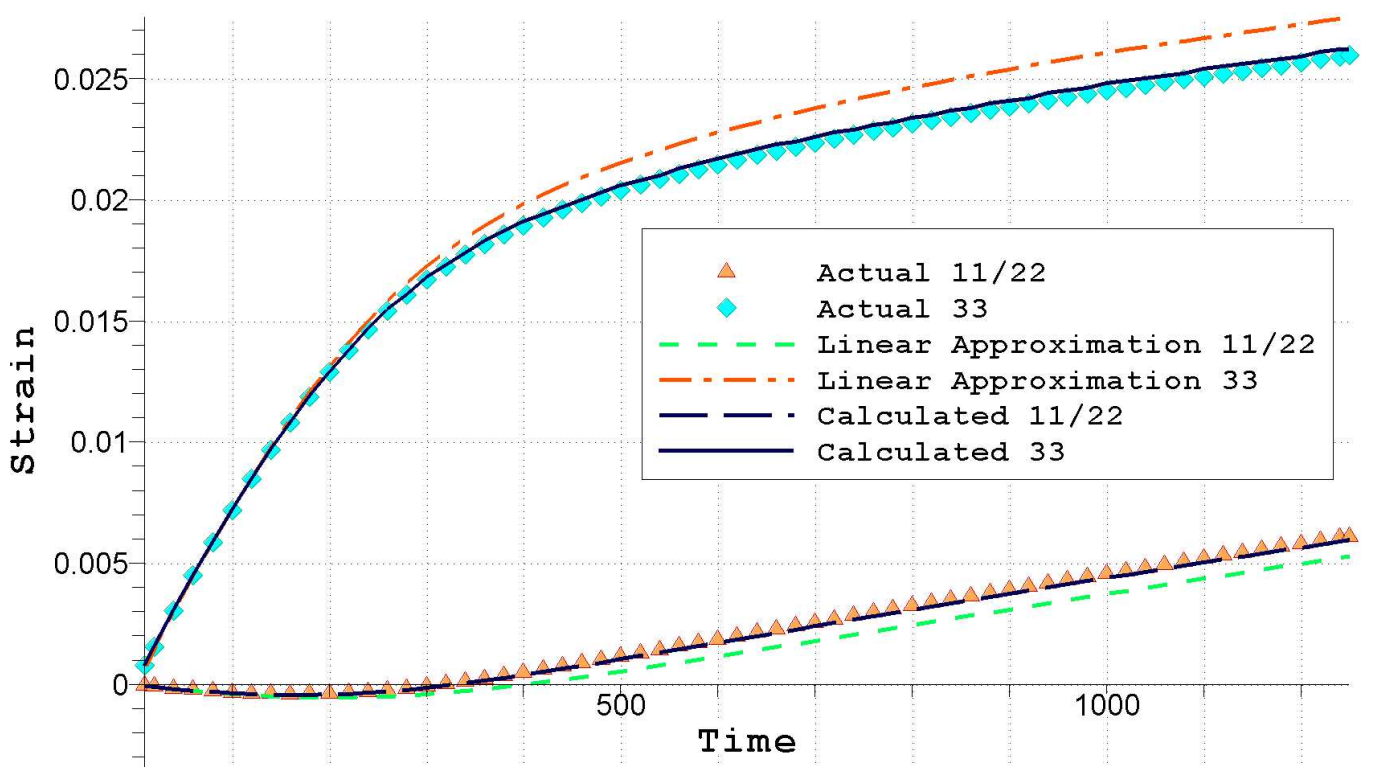

Figure 3: Plot showing strain against time. 
A high degree of accuracy is achieved using the proposed algorithm to calculate the viscosity and elastic strain.

\section{Conclusion}

A theory is proposed which allows the prediction of elastic strain and viscosity before any explicit definition of nonlinear functions are chosen. This ensures objectivity of the calculations to actual, rather than best fit, results that may occur during more rigid parameter estimation.

The high accuracy of the prediction of simulation results justifies the proposed theory and shows it to be a highly effective means to carry out both parameter estimation and material response research.

Acknowledgements The authors acknowledge Gough Plastic Pty Ltd for their financial support towards this research.

\section{References}

[1] A. Ben-Israel and T. N. E. Greville. Generalized inverses: Theory and applications. Cambridge University Press, Sydney, 1974. C655

[2] J. Bonet and R. D. Wood. Nonlinear continuum mechanics for finite element analysis. Cambridge University Press, Melbourne, 1997. C648, C653

[3] N. Brusselle-Dupend, D. Lai, X. Feaugas, M. Guigon, and M. Clavel. Mechanical behavior of a semicrystalline polymer before necking. Part I: Characterization of uniaxial behavior. Polym. Eng. Sci., 41:66-76, 2001. doi:10.1002/pen.10709. C647, C649, C656 
[4] N. Brusselle-Dupend, D. Lai, X. Feaugas, M. Guigon, and M. Clavel. Mechanical behavior of a semicrystalline polymer before necking. Part II: Modeling of uniaxial behavior. Polym. Eng. Sci., 43:501-518, 2003. doi:10.1002/pen.10041. C647, C649, C656

[5] M. Ortiz, P. M. Pinsky, and R. L. Taylor. Operator split methods for the numerical solution of the elastoplastic dynamic problem. Comput. Methods Appl. Mech. Engrg., 39:137-157, 1983. doi:10.1016/0045-7825(83)90018-X. C651

[6] R. Penrose. A generalized inverse for matrices. Proc. Cambridge Philos. Soc., 51:406-413, 1955. C655

[7] D. Perić. On a class of consitutive equations in viscoplasticity: Formulation and computational issues. Int. J. Numer. Meth. Engng., 36:1365-1393, 1993. doi:10.1002/nme.1620360807. C652

[8] S. Reese and S. Govindjee. A theory of finite viscoelasticity and numerical aspects. Int. J. Solids Structures, 35:3455-3482, 1998a. doi:10.1016/S0020-7683(97)00217-5. C648, C649

[9] J. C. Simo and T. J. R. Hughes. Computational Inelasticity. Springer, New York, 2000. C647, C648, C652

[10] J. C. Simo and R. L. Taylor. Quasi-incompressible finite elasticity in principal stretches. continuum basis and numerical algorithms.

Comput. Methods Appl. Mech. Engrg., 85:273-310, 1991. doi:10.1016/0045-7825(91)90100-K. C648

[11] E. H. Twizell and R. W. Ogden. Non-linear optimization of the material constants in Ogden's stress-deformation function for incompressible isotropic elastic materials. J. Austral. Math. Soc. Ser. B, 24:424-434, 1983. C646 


\section{Author addresses}

1. D. W. Holmes, School of Engineering, James Cook University, Townsville, Australia.

mailto:david.holmes1@jcu.edu.au

2. J. G. Loughran, School of Engineering, James Cook University, Townsville, Australia. 